\title{
Sandwich-like layer-by-layer assembly of gold nanoparticles with tunable SERS properties
}

\author{
Zhicheng Liu ${ }^{* 1}$, Lu Bai ${ }^{2}$, Guizhe Zhao ${ }^{1}$ and Yaqing Liu ${ }^{* 1}$
}

\author{
Letter \\ Address: \\ ${ }^{1}$ Shanxi Province Key Laboratory of Functional Nanocomposites, \\ School of Materials Science and Engineering, North University of \\ China, Taiyuan 030051, China and ${ }^{2}$ School of Chemical and \\ Environmental Engineering, North University of China, Taiyuan \\ 030051, China \\ Email: \\ Zhicheng Liu* - zcliu11@gmail.com; Yaqing Liu* - zfflyq98@163.com \\ * Corresponding author \\ Keywords: \\ assembly; layer-by-layer; multilayer thin film; nanoparticle; \\ polyelectrolyte
}

\begin{abstract}
doi:10.3762/bjnano.7.95
Received: 11 March 2016

Accepted: 26 June 2016

Published: 15 July 2016
\end{abstract}

Beilstein J. Nanotechnol. 2016, 7, 1028-1032.

This article is part of the Thematic Series "Nanostructures for sensors, electronics, energy and environment III".

Guest Editor: N. Motta

(c) 2016 Liu et al.; licensee Beilstein-Institut.

License and terms: see end of document.

\begin{abstract}
Sandwich-like layer-by-layer thin films consisting of polyelectrolytes and gold nanoparticles were utilized to construct surface-enhanced Raman scattering (SERS) substrates with tunable SERS properties. It is found that both the size of the nanoparticles in the layers and the interlayer distance significantly influence the SERS performance of the multilayered thin film. These simple, lowcost, easily processable and controllable SERS substrates have a promising future in the field of molecular sensing.
\end{abstract}

\section{Introduction}

Surface-enhanced Raman scattering (SERS) spectroscopy, which relies on metal nanostructures made of noble metals $(\mathrm{Au}$, $\mathrm{Ag}$ and $\mathrm{Cu}$ ) that sustain localized surface plasmon resonance (LSPR), is applied as a promising analytical tool for detecting and identifying trace amounts of molecular species [1-3]. The fabrication of excellent SERS substrates using simple and low-cost methods is currently an attractive topic in this field [4]. Engineering metal nanoparticle assemblies with tunable plasmonic coupling properties shows high potential for that purpose [5].

Among various top-down and bottom-up techniques, layer-bylayer $(\mathrm{LbL})$ assembly is a facile and cost-efficient way for the controllable deposition of numerous components [6-8]. Multilayer nanostructures with complex morphologies and functions could be prepared conveniently through the LbL assembly process, which is mainly driven by electrostatic interaction. Especially, multilayer thin films consisting of polymers and metal nanoparticles (NPs) have been extensively explored, and show interesting optical and SERS properties [9-14]. Wang and Dong et al. reported that polyelectrolyte-gold nanorod multilayer thin films could be obtained using LbL assembly techniques via electrostatic interactions [15]. By controlling the number of deposition layers, the plasmonic properties as well as the SERS properties could be tuned easily. Moreover, Kim and co-workers showed that gold nanoparticles (Au NPs) could be 
assembled onto polyelectrolyte multilayer films and act as seeds for the following NP growth [16]. The grown NP films were demonstrated to be stable and reproducible SERS substrates. In addition to assembling only one type of NPs, Zhang et al. fabricated bimetallic gold-silver multilayer films by alternating the adsorption of polyethyleneimine-silver ions and Au NPs onto substrates and the subsequent in situ reduction of the silver ions [17]. Compared with the parallel samples, the bimetallic LbL film showed improved SERS properties. Although a few examples of SERS substrates based on LbL strategy have been given, the design and engineering of such SERS substrates is still challenging, and the probing of the structure-dependent SERS performance remains a considerable issue.

Recently, we have shown that highly reproducible and stable SERS substrates could be obtained via LbL assembly of polyelectrolyte and Au NPs [18]. The tuning of SERS intensities was realized by varying the number of deposited Au NP layer. Here, we present that SERS properties of LbL thin film could be controlled by assembling Au NPs of different sizes or changing the interlayer distance between Au NP layers. Sandwich-like LbL thin films with three bilayers, which were fabricated by alternating deposition of polyelectrolytes and Au NPs, are designed to explore the relationship between multilayer nanostructures and SERS performance.

\section{Experimental}

All chemicals such as poly(diallyldimethylammonium chloride) (PDDA, $\left.M_{\mathrm{w}}=200,000-350,000\right)$, poly(sodium 4-styrenesulfonate) (PSS, $M_{\mathrm{w}}=70000$ ), and 4-aminothiophenol (4-ATP) were obtained from Sigma-Aldrich and used without further treatment. Negatively charged citrate-stabilized Au NPs were prepared using the classic Turkevich method [19-21]. Briefly, a $50 \mathrm{~mL}$ aqueous solution that contained $0.5 \mathrm{~mL} 1 \mathrm{wt} \%$ aqueous $\mathrm{HAuCl}_{4}$ solution was heated to boil under gentle stirring. Then, a certain amount of $1 \mathrm{wt} \%$ sodium citrate solution was added quickly. The obtained red-wine colored NP solution was stored at $4{ }^{\circ} \mathrm{C}$ and used for the LbL assembly. Hydrophilic quartz slides were used to deposit the first PDDA layer. Sandwich-like $\mathrm{LbL}$ thin films were obtained by alternative immersion of PDDA ( $1 \mathrm{mg} / \mathrm{mL}$, with $1.5 \mathrm{M} \mathrm{NaCl}, 30 \mathrm{~min}$ ) and Au NP solution $(12 \mathrm{~h})$. After each immersion step, the thin film was rinsed with water and dried under $\mathrm{N}_{2}$. Since only two different sizes of $\mathrm{Au}$ NPs were utilized, four kinds of sandwich-like LbL thin films consisting of NP layers of different NP sizes were achieved. The LbL thin film with three layers of small Au NPs is marked as SSS, while the one with three layers of big Au NPs is marked as BBB. Similarly, two other kinds of thin films, namely SBS and BSB, were also obtained. In order to control the interlayer distance between the Au NP layers, different numbers of PDDA/PSS bilayers were inserted into the sand- wich-like nanostructure to separate the NP layers. Before SERS measurements, $50 \mu \mathrm{L}$ of $0.1 \mathrm{mM} 4$-ATP ethanol solution was dropped on the as-prepared substrate and left to dry in the air.

The as-synthesized Au NP solution was characterized by UV-visible (UV-vis) spectroscopy (Cary 5000). Field emission scanning electron microscopy (FE-SEM, Hitachi S-4800) and transmission electron microscopy (TEM, JEOL JEM 1011) were used to image the LbL thin films and the NPs, respectively. SERS spectra excited at $1064 \mathrm{~nm}$ were recorded with a Thermo Nicolet FT-Raman 960 spectrometer at a power of $200 \mathrm{~mW}$. All the spectra were collected by averaging 1024 scans.

\section{Results and Discussion}

$\mathrm{Au}$ NPs are regarded as certified and promising nanoscale building blocks for both LbL assembly and SERS substrates. Two different sizes of Au NPs were synthesized by simple control of the added volume of sodium citrate solution. Small $\mathrm{Au}$ NPs $(17.0 \pm 1.2 \mathrm{~nm})$ were obtained when $2 \mathrm{~mL}$ sodium citrate solution was injected, while big Au NPs $(42.9 \pm 5.4 \mathrm{~nm})$ were obtained when $0.65 \mathrm{~mL}$ sodium citrate solution was injected, as shown in Figure 1. The optical absorption peaks located at 520 and $532 \mathrm{~nm}$ indicate the strong surface plasmon resonance of the NPs. These uniform negatively charged citrateprotected Au NPs could be readily used in electrostatic LbL assembly.

Typical sandwich-like multilayer thin films were prepared by sequential deposition of polyelectrolytes and Au NPs. For example, the BSB thin film formed after the substrate was alternately dipped into PDDA/big Au NP/PDDA/small Au NP/ PDDA/big Au NP solutions. From the point of view of Au NPs, these sandwich-like nanostructures are ideal for the evaluation of NP size effects on the SERS performance of NP assemblies. Figure 2 presents the SEM images of the SSS, SBS, BSB and BBB multilayer thin films. Undoubtedly, both small and big Au NPs were assembled into the thin films, and the NP size did not change during the assembly process. It is clear that the sandwich-like LbL assembly of Au NPs occurs via lateral expansion mode, which is consistent with previous results $[18,22,23]$. Interestingly, compared with the BSB thin film, there were more small Au NPs in the SBS thin film, though the number of big Au NPs was about the same. This phenomenon may result from the steric hindrance of the pre-assembled big Au NPs. In other words, the first big NP layer in the BSB thin film is more unfavorable for the following deposition of small NPs, resulting in less small NPs in the non-stratified thin film. What is more, these LbL thin films are relatively uniform (Figure S1, Supporting Information File 1), which is important for reproducible SERS performance [18]. 


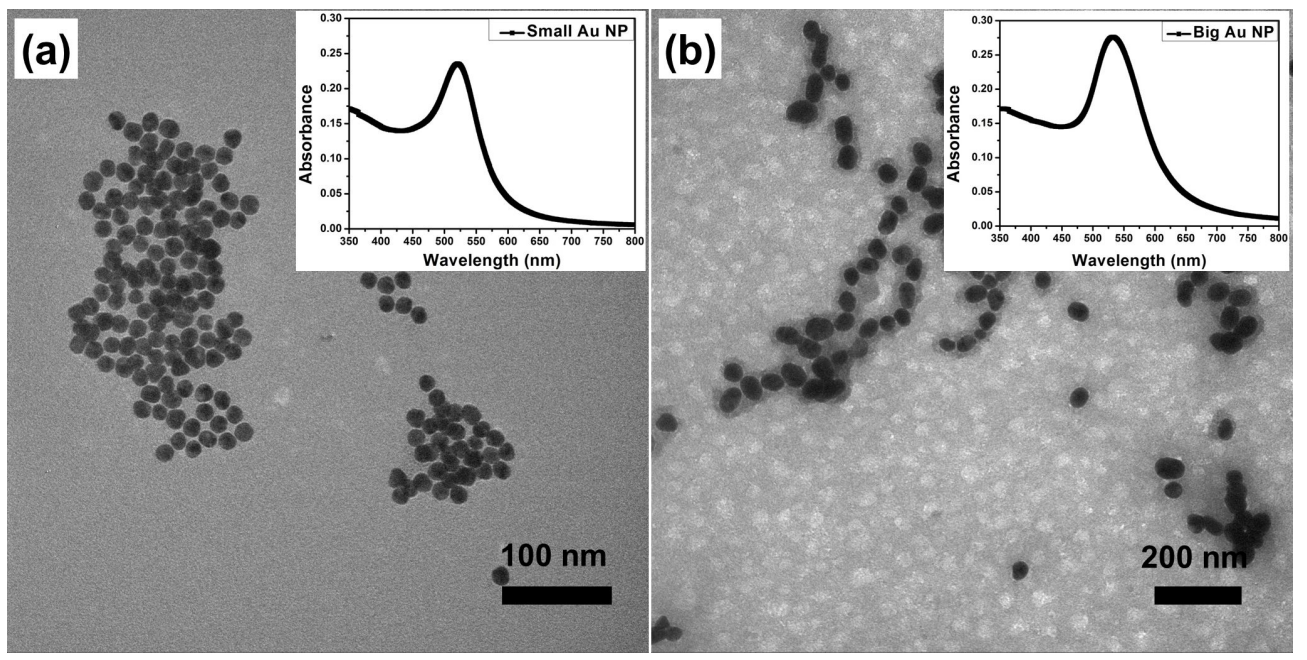

Figure 1: TEM images of the obtained Au NPs: (a) small Au NP, (b) big Au NP. The insets show the UV-vis spectra of the corresponding Au NP solutions.
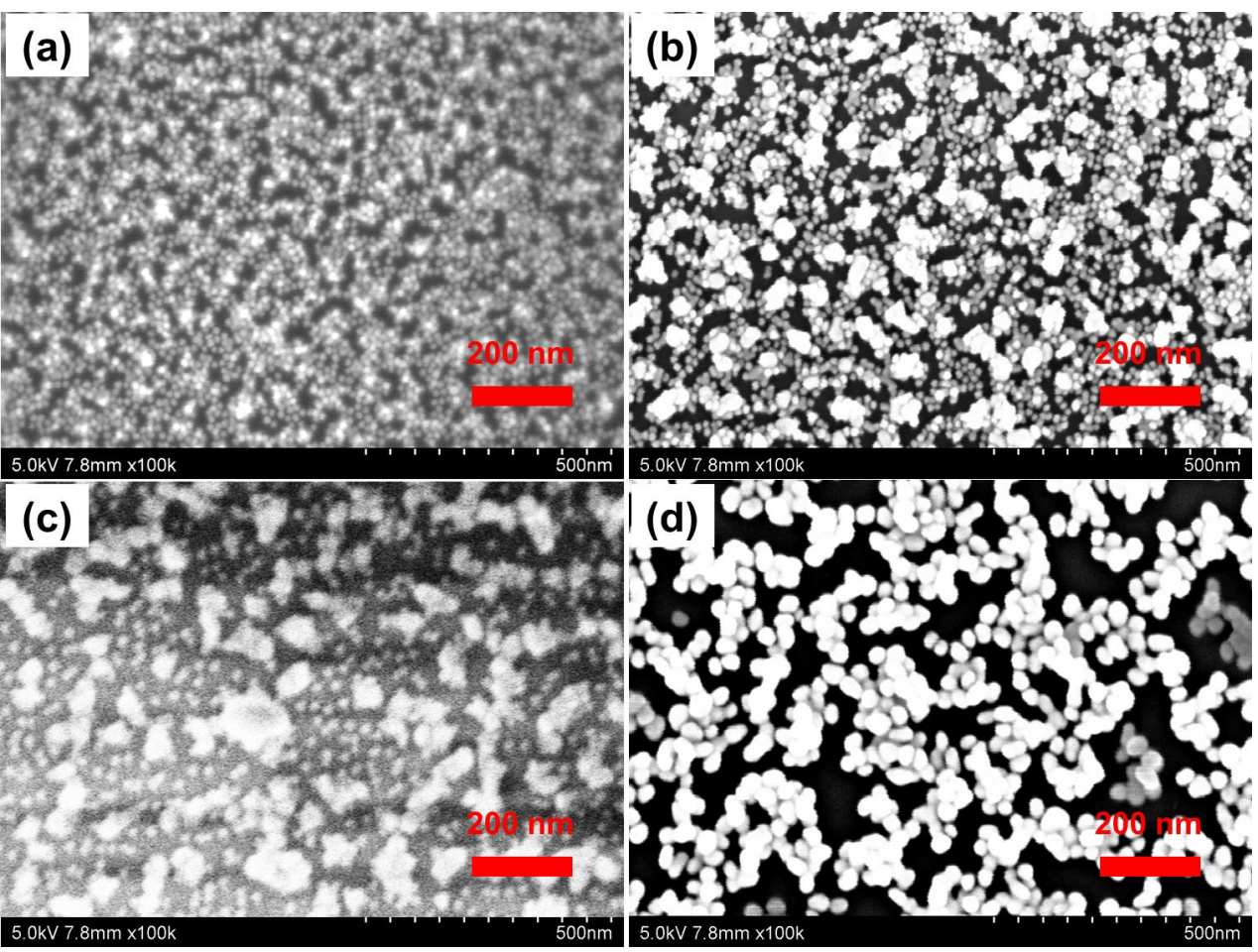

Figure 2: SEM images of multilayer thin films consisted of PDDA and Au NPs of different sizes: (a) SSS, (b) SBS, (c) BSB, (d) BBB.

In order to evaluate the SERS performance of the sandwich-like nanostructures, 4-ATP was chosen as SERS probing molecule because of its well-defined Raman vibrational signatures [24]. Figure 3a shows the SERS spectra of the corresponding multilayer thin films. It is noted that the spectra are dominated by the $\mathrm{a}_{1}$ vibration modes, which show distinct peaks at $1587 \mathrm{~cm}^{-1}$ $\left(v_{\mathrm{C}-\mathrm{C}}\right)$ and $1078 \mathrm{c}^{-1}\left(v_{\mathrm{C}-\mathrm{S}}\right)$. It suggests that the electromagnetic field enhancement dominates the SERS performance [15]. The relative SERS intensities of the films follow the order: BBB $>$ SBS $>$ BSB $>$ SSS. Moreover, the actual SERS performance, which is described by the enhancement factors (EF) of these multilayer thin films, was calculated using the equation $\mathrm{EF}=\left(I_{\mathrm{SERS}} / N_{\text {ads }}\right) /\left(I_{\text {bulk }} / N_{\text {bulk }}\right)$, where $I_{\text {SERS }}$ and $I_{\text {bulk }}$ are the intensity of a vibrational mode in the SERS spectrum and bulk 

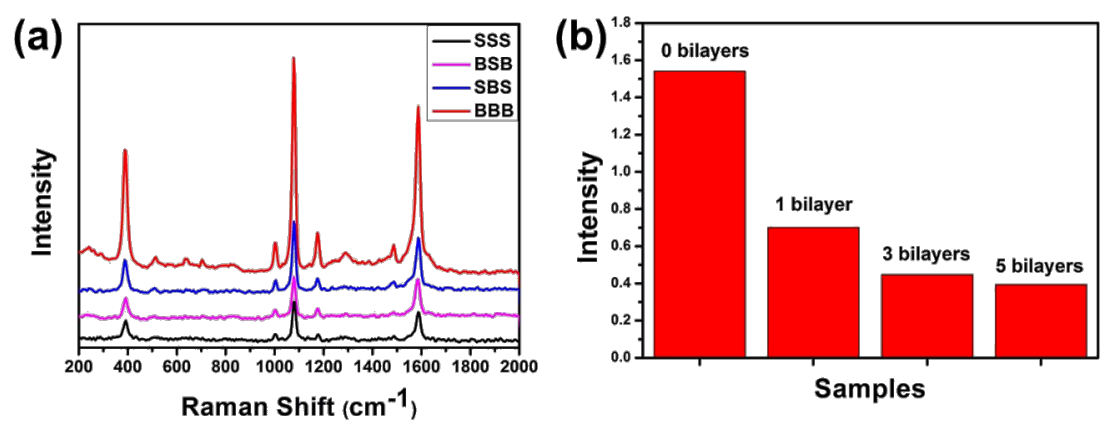

Figure 3: (a) SERS spectra of the sandwich-like multilayer thin films; (b) SERS intensity variations at $1078 \mathrm{~cm}^{-1}$ for LbL thin films inserted with different numbers of PDDA/PSS bilayers.

sample, and $N_{\text {ads }}$ and $N_{\text {bulk }}$ are the number of molecules adsorbed on the SERS substrate and bulk molecules excited by the laser, respectively. Using the $1587 \mathrm{~cm}^{-1}$ band, the EF values for the SSS, SBS, BSB and BBB thin films are calculated to be about $5.9 \times 10^{4}, 5.4 \times 10^{4}, 5.0 \times 10^{4}$ and $3.6 \times 10^{4}$, respectively (see Supporting Information File 1 for the detailed calculation). It is known that SERS activity is impacted by multiple factors such as size, shape and interparticle coupling of NPs. Compared with the BBB thin film, the increase of the enhancement factor of the SSS thin film could be primarily ascribed to electromagnetic enhancement of increased NP numbers, since there might be more hot-spots in the SSS thin film $[25,26]$. As shown in Figure $2 \mathrm{~b}$ and Figure 2c, more hot-spots would also be generated for the SBS thin film, resulting in a better SERS performance than the BSB thin film. Though NP size was demonstrated to greatly impact the SERS performance, it is difficult to obtain dense large NPs layer using the LbL assembly, which is critical for the improved electromagnetic enhancement.

It has been shown that the distance between adjacent NP layers has notable effects on the optical, magnetic and electrochemical properties of LbL NP thin films [27-33]. However, this interesting effect on SERS properties is almost unexplored [34]. Since the interparticle distance may strongly influence the SERS performance, PDDA/PSS bilayers were introduced to separate the neighboring NP layers. Figure $3 \mathrm{~b}$ displays the SERS intensities at $1078 \mathrm{~cm}^{-1}$ for sandwich-like thin films inserted with different numbers of PDDA/PSS bilayers. Obviously, the SERS intensity decreased significantly with increasing number of separating PDDA/PSS bilayers. This is possibly because localized surface plasmon from Au NPs of neighboring layer was not coupled intensively, after the interlayer gap was broadened by inserting bilayers [35]. It is noteworthy that NP layers separated by three polyelectrolyte monolayer provided the strongest SERS signals, as recently presented by Carsten Rockstuhl and co-workers [34]. Nevertheless, the fact that the multilayer NP thin film without separating layers pos- sesses the strongest SERS performance is reasonable, because the thickness of one polyelectrolyte monolayer assembled at high salt concentration ( $1.5 \mathrm{M} \mathrm{NaCl}$, this work) may be similar to that of three polyelectrolyte monolayers assembled at low salt concentration (0.1 M NaCl, work of Rockstuhl et al.) [36]. Overall, controlling the interlayer distance of the NP layers is another efficient way to tune the SERS properties of multilayer thin films.

\section{Conclusion}

Sandwich-like LbL assemblies of Au NPs were designed as model SERS substrate. The SERS performance could be readily tuned by using Au NPs of different sizes or introducing insert layers with controllable thickness. The methods and strategies involved in this work are rather simple. The fabricated SERS substrates may pave the way for highly efficient and sensitive sensing of small molecules.

\section{Supporting Information}

\section{Supporting Information File 1}

Additional experimental data.

[http://www.beilstein-journals.org/bjnano/content/ supplementary/2190-4286-7-95-S1.pdf]

\section{Acknowledgements}

This work was supported by National Natural Science Foundation of China (Grant 21444005, 21504083 and 21505123) and Scientific and Technological Innovation Programs of Higher Education Institutions in Shanxi.

\section{References}

1. Schlücker, S. Angew. Chem., Int. Ed. 2014, 53, 4756-4795. doi:10.1002/anie.201205748

2. Moskovits, M. Phys. Chem. Chem. Phys. 2013, 15, 5301-5311. doi:10.1039/c2cp44030j 
3. Pieczonka, N. P. W.; Aroca, R. F. Chem. Soc. Rev. 2008, 37, 946-954. doi:10.1039/b709739p

4. Ko, H.; Singamaneni, S.; Tsukruk, V. V. Small 2008, 4, 1576-1599. doi:10.1002/smll.200800337

5. Tong, L.; Zhu, T.; Liu, Z. Chem. Soc. Rev. 2011, 40, 1296-1304. doi:10.1039/C001054P

6. Jiang, C.; Tsukruk, V. V. Adv. Mater. 2006, 18, 829-840. doi:10.1002/adma.200502444

7. Borges, J.; Mano, J. F. Chem. Rev. 2014, 114, 8883-8942. doi:10.1021/cr400531v

8. Richardson, J. J.; Björnmalm, M.; Caruso, F. Science 2015, 348, 411 doi:10.1126/science.aaa2491

9. Abalde-Cela, S.; Ho, S.; Rodríguez-González, B.; Correa-Duarte, M. A.; Álvarez-Puebla, R. A.; Liz-Marzán, L. M.; Kotov, N. A. Angew. Chem., Int. Ed. 2009, 48, 5326-5329. doi:10.1002/anie.200901807

10. Bao, Y.; Vigderman, L.; Zubarev, E. R.; Jiang, C. Langmuir 2012, 28, 923-930. doi:10.1021/la203993m

11. Tian, R.; Li, M.; Teng, H.; Luo, H.; Yan, D.; Wei, M. J. Mater. Chem. C 2015, 3, 5167-5174. doi:10.1039/C4TC02714K

12. Aoki, P. H. B.; Alessio, P.; De Saja, J. A.; Constantino, C. J. L. J. Raman Spectrosc. 2010, 41, 40-48. doi:10.1002/jrs.2415

13. dos Santos, D. S.; Goulet, P. J. G.; Pieczonka, N. P. W.; Oliveira, O. N.; Aroca, R. F. Langmuir 2004, 20, 10273-10277. doi:10.1021/la048328j

14. Zhang, F.; Srinivasan, M. P. J. Colloid Interface Sci. 2008, 319, 450-456. doi:10.1016/j.jcis.2007.12.008

15. Hu, X.; Cheng, W.; Wang, T.; Wang, Y.; Wang, E.; Dong, S. J. Phys. Chem. B 2005, 109, 19385-19389. doi:10.1021/jp052706r

16. Koo, H. Y.; Choi, W. S.; Park, J.-H.; Kim, D.-Y. Macromol. Rapid Commun. 2008, 29, 520-524. doi:10.1002/marc.200700683

17. Zhang, L.; Wang, C.; Zhang, Y. Appl. Surf. Sci. 2012, 258, 5312-5318. doi:10.1016/j.apsusc.2012.01.101

18. Liu, Z.; Yan, Z.; Bai, L. Appl. Surf. Sci. 2016, 360, 437-441. doi:10.1016/j.apsusc.2015.09.151

19. Enustun, B. V.; Turkevich, J. J. Am. Chem. Soc. 1963, 85, 3317-3328. doi:10.1021/ja00904a001

20. Liu, Z.; Chang, T.; Huang, H.; He, T. RSC Adv. 2013, 3, 20464-20470. doi:10.1039/c3ra43394c

21. Liu, Z.; Chang, T.; Huang, H.; He, T. ACS Appl. Mater. Interfaces 2015 , 7, 25938-25945. doi:10.1021/acsami.5b08751

22. Ostrander, J. W.; Mamedov, A. A.; Kotov, N. A. J. Am. Chem. Soc. 2001, 123, 1101-1110. doi:10.1021/ja0029578

23. Yuan, W.; Li, C. M. Langmuir 2009, 25, 7578-7585. doi:10.1021/la901443x

24. Huang, Y.-F.; Wu, D.-Y.; Zhu, H.-P.; Zhao, L.-B.; Liu, G.-K.; Ren, B.; Tian, Z.-Q. Phys. Chem. Chem. Phys. 2012, 14, 8485-8497. doi:10.1039/c2cp40558j

25. Fang, P.-P.; Li, J.-F.; Yang, Z.-L.; Li, L.-M.; Ren, B.; Tian, Z.-Q. J. Raman Spectrosc. 2008, 39, 1679-1687. doi:10.1002/jrs.2066

26. Joseph, V.; Matschulat, A.; Polte, J.; Rolf, S.; Emmerling, F.; Kneipp, J. J. Raman Spectrosc. 2011, 42, 1736-1742. doi:10.1002/jrs.2939

27. Schmitt, J.; Decher, G.; Dressick, W. J.; Brandow, S. L.; Geer, R. E.; Shashidhar, R.; Calvert, J. M. Adv. Mater. 1997, 9, 61-65. doi:10.1002/adma.19970090114

28. Jiang, G.; Baba, A.; Ikarashi, H.; Xu, R.; Locklin, J.; Kashif, K. R.; Shinbo, K.; Kato, K.; Kaneko, F.; Advincula, R. J. Phys. Chem. C 2007, 111, 18687-18694. doi:10.1021/jp075986e
29. Vial, S.; Pastoriza-Santos, I.; Pérez-Juste, J.; Liz-Marzán, L. M. Langmuir 2007, 23, 4606-4611. doi:10.1021/la063753t

30. Kiel, M.; Mitzscherling, S.; Leitenberger, W.; Santer, S.; Tiersch, B.; Sievers, T. K.; Möhwald, H.; Bargheer, M. Langmuir 2010, 26, 18499-18502. doi:10.1021/la103609f

31. Chaikin, Y.; Leader, H.; Popovitz-Biro, R.; Vaskevich, A.; Rubinstein, I. Langmuir 2011, 27, 1298-1307. doi:10.1021/la103913u

32. Pichon, B. P.; Louet, P.; Felix, O.; Drillon, M.; Begin-Colin, S.; Decher, G. Chem. Mater. 2011, 23, 3668-3675. doi:10.1021/cm201139s

33. Schmidt, A. R.; Nguyen, N. D. T.; Leopold, M. C. Langmuir 2013, 29 , 4574-4583. doi:10.1021/la400359x

34. Mühlig, S.; Cialla, D.; Cunningham, A.; März, A.; Weber, K.; Bürgi, T.; Lederer, F.; Rockstuhl, C. J. Phys. Chem. C 2014, 118, 10230-10237. doi:10.1021/jp409688p

35. Yi, Z.; Yi, Y.; Luo, J.; Ye, X.; Wu, P.; Ji, X.; Jiang, X.; Yi, Y.; Tang, Y. RSC Adv. 2015, 5, 1718-1729. doi:10.1039/C4RA06141A

36. McAloney, R. A.; Sinyor, M.; Dudnik, V.; Goh, M. C. Langmuir 2001, 17, 6655-6663. doi:10.1021/la010136q

\section{License and Terms}

This is an Open Access article under the terms of the Creative Commons Attribution License (http://creativecommons.org/licenses/by/2.0), which permits unrestricted use, distribution, and reproduction in any medium, provided the original work is properly cited.

The license is subject to the Beilstein Journal of

Nanotechnology terms and conditions:

(http://www.beilstein-journals.org/bjnano)

The definitive version of this article is the electronic one which can be found at:

doi:10.3762/bjnano.7.95 